\title{
Deuteron breakup at zero angle in the Coulomb nuclear field
}

\author{
Igor Sitnik $^{1, *}$ \\ ${ }^{1}$ Joint Institute for Nuclear Research, Dubna, Russia
}

\begin{abstract}
Deuteron breakup cross sections on the $\mathrm{C}$ and $\mathrm{CH}_{2}$ targets have been measured up to the proton internal momenta of $0.3 \mathrm{GeV} / \mathrm{c}$. The cross-sections ${ }^{12} C(d, p) X$ and ${ }^{1} H(d, p) X$ reactions have been obtained with high precision. The obtained data are compared with previous measurements.

The behavior features in the vicinity of the cross section maximum were studied in dependence on the transversal momentum in the region of $0.01<p_{t}<$ $0.16 \mathrm{GeV} / \mathrm{c}$.

The measurements have been performed at the Veksler Baldin Laboratory of High Energy Physics of the Joint Institute for Nuclear Research.
\end{abstract}

\section{Introduction}

Disintegration of the lightest nuclei is one of effective tools in studying the few-body problem. The first measurements of the ${ }^{12} C(d, p) X$ in the full range of the internal proton momenta were fulfilled in 1983 [1]. The measurements were repeated with a higher precision on the $C$ and $\mathrm{CH}_{2}$ targets in 1992 [2].

Here we present the results of the new measurements on the $\mathrm{C}$ and $\mathrm{CH}_{2}$ targets, which differ from the previous ones by:

- high resolution measurement of all momentum components of secondary protons;

- large angle-momentum acceptance for secondary protons;

- huge statistics in the momentum range of secondary protons of $0-0.3 \mathrm{GeV} / \mathrm{c}$.

\section{Experiment}

The setup "Strela" [3] was located on the extracted beam line of the Nuclotron. The primary deuteron beam with momentum of $3.5 \mathrm{GeV} / \mathrm{c}$ had the intensity of $10^{6}$, which provided the opportunity of measuring input particle tracks. The bending angle in the magnet was of 0.289 rad. The scintillator counters were used for triggers. The track parameters were measured by drift chambers. Their space accuracy was about $0.05 \mathrm{~mm}$. The momentum resolution and efficiency of the setup was of $0.9 \%$ and $(0.91 \pm 1) \%$, respectively.

The program package [6] was used for the track reconstruction and offsets adjusting.

\footnotetext{
*e-mail: sitnik@jinr.ru
} 


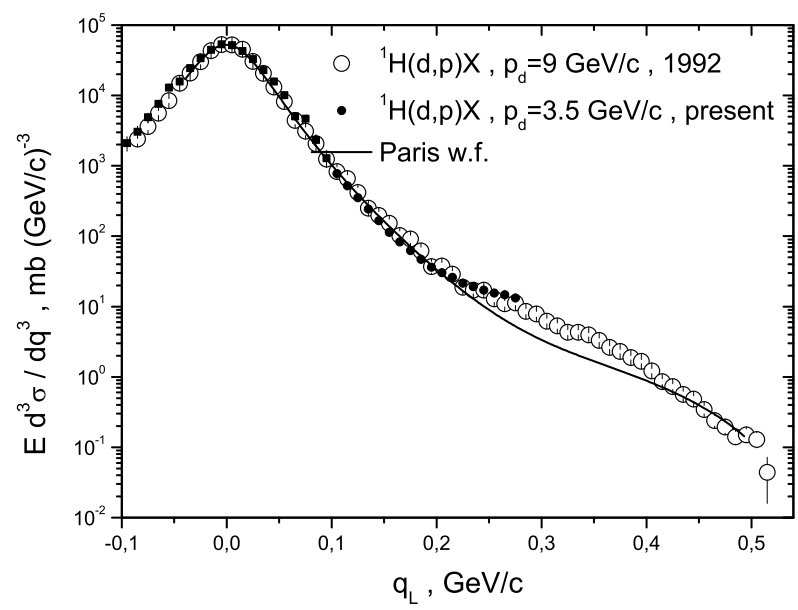

Figure 1. Invariant differential cross-section ${ }^{1} H(d, p) X$

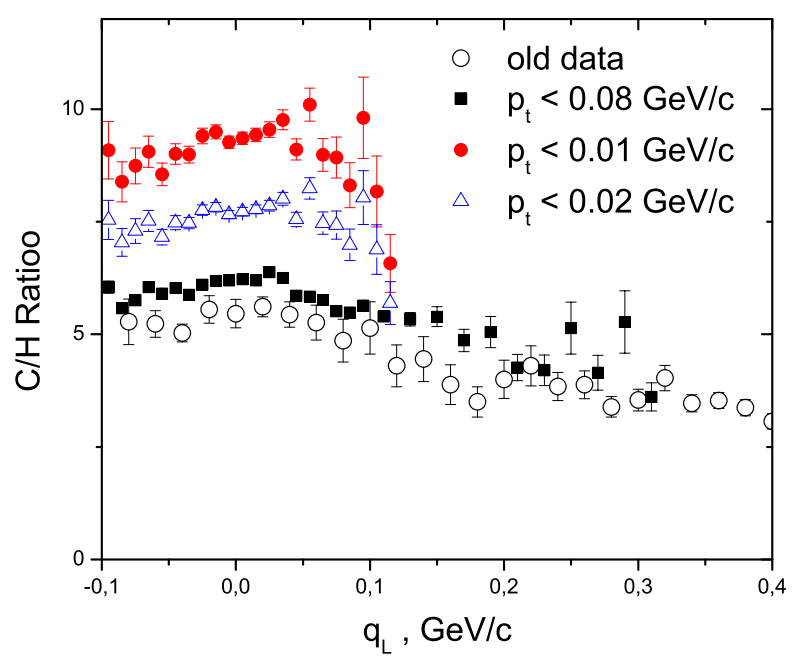

Figure 2. ${ }^{12} C(d, p) X /{ }^{1} H(d, p) X$ ratios at different transversal momentum regions

\section{Results}

In. Fig. 1 the presented data are compared with results of [2]. To provide conditions of the experiment [2], the restriction on transversal momentum $p_{t} \leq 0.08 \mathrm{GeV} / \mathrm{c}$ was used for this picture. Here $q_{L}$ is a longitudinal momentum of the proton in the deuteron rest frame. Firstly this result was presented in [4]. In spite of essentially different momenta of projectiles, a good agreement between two sets of data takes place, including the region of the anomalous 


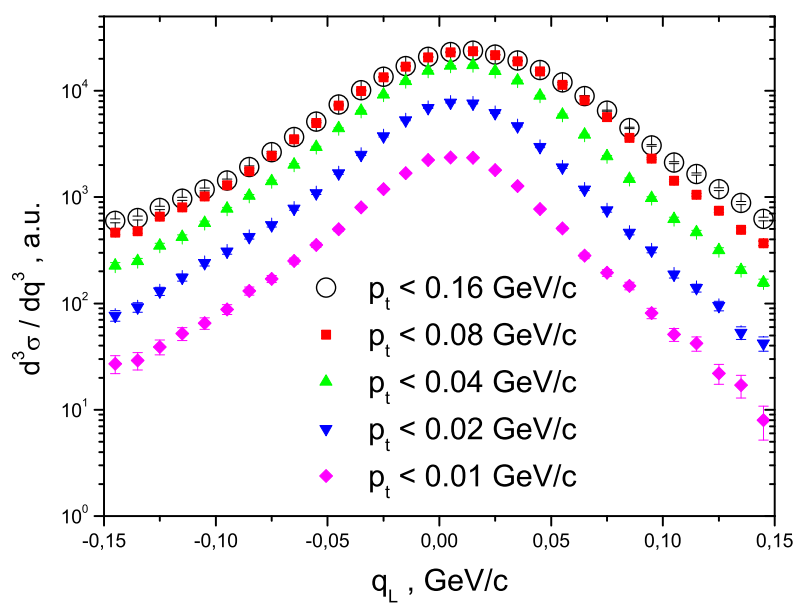

Figure 3. ${ }^{12} C(d, p) X$ spectra in different $p_{t}$-corridors

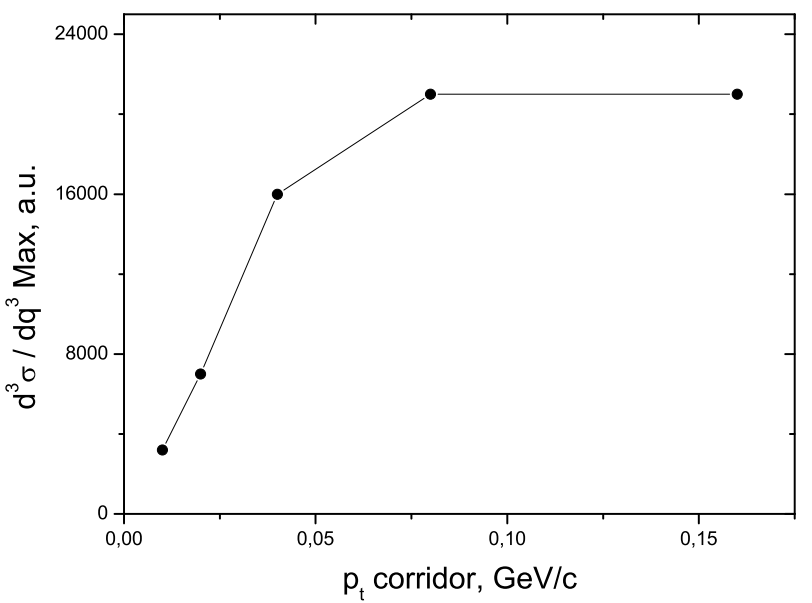

Figure 4. Maxima of ${ }^{12} C(d, p) X$ spectra in dependence on $p_{t}$-corridor

behavior of the cross sections in the vicinity of $0.3 \mathrm{GeV} / \mathrm{c}$. A different reaction mechanism, offered to explain the anomaly, like [5], has the energy dependence and so, is not valid. We deal with the deuteron structure effect.

In this paper the behavior of spectra in different $p_{t}$-corridors was studied. In Fig. 2 we demonstrate the ratio of cross-sections for ${ }^{12} C(d, p) X$ and ${ }^{1} H(d, p) X$ reactions. Increase in the ratio with decreasing $p_{t}$ at $q_{L} \simeq 0$ is easy to explain that at the smallest $p_{t}$ the Coulomb interaction prevails. Then, let us study the behavior of ${ }^{12} C(d, p) X$ spectra in different $p_{t^{-}}$ corridors. In Fig. 3 these spectra are shown at $\left|q_{L}\right|<0.15 \mathrm{GeV} / \mathrm{c}$. In Fig. 4 the dependence 
of maxima for each spectrum on $p_{t}$-corridor value is presented. It is easy to suppose that at $p_{t}>0.08 \mathrm{GeV} / \mathrm{c}$ the strong interaction predominates, but at $p_{t}<0.04 \mathrm{GeV} / \mathrm{c}$ the Coulomb disintegration of the deuteron takes place.

\section{Conclusions}

- Breakup cross-sections show energy independence in the GeV region.

- The Coulomb disintegration of the deuteron has discovered.

Acknowledgment. This work was supported under Slovak Grant Agency VEGA No. 1/0113/18.

\section{References}

[1] V. G. Ableev et al., Nucl.Phys. A 393, 491 (1983)

[2] V. G. Ableev et al., JINR Rapid. Com. 1[52]-92, 10 (1992)

[3] V. V. Glagolev et al., Instr. and Experim. techn. 56, 387 (2013)

[4] S.N. Basylev et al., in The XXII International Baldin Seminar on High Energy Physics Problems, JINR, Dubna, 2014, PoS Baldin ISHEPP XXII, 034 (2014)

[5] A. P. Ierusalimov, G. I. Lykasov, M. Viviani, arXiv:1002.0249 (2010)

[6] I. M. Sitnik, Comput.Phys.Commun. 185, 599 (2014). ibid. 209, 199 (2016)

[7] A. P. Kobushkin, Ya. D. Krivenko-Emetov, Ukr. J. Phys. 53(8), 751 (2008)

[8] G. Faldt, Nucl. Phys. B 43, 591 (1972) 